\title{
Jet Correlations of Identified Particles in PHENIX
}

\author{
Anne Sickles $\dagger$ for the PHENIX Collaboration $\S$ \\ $\dagger$ Department of Physics and Astronomy, State University of New York at Stony \\ Brook, Stony Brook NY 11794-3800, USA
}

\begin{abstract}
Azimuthal two particle correlations at intermediate $p_{T}$ with one of the particles identified have been measured at PHENIX. Trigger $\left(2.5<p_{T}<4.0 \mathrm{GeV} / \mathrm{c}\right)$ baryons and mesons show little significant difference in the number of associated particles $\left(1.7<p_{T}<2.5 \mathrm{GeV} / \mathrm{c}\right)$ independent of centrality. For inclusive hadron triggers with $2.5<p_{T}<4.0 \mathrm{GeV} / \mathrm{c}$, associated fragmentation particles with $1.0<$ $p_{T}<2.5 \mathrm{GeV} / \mathrm{c}$ show a higher baryon to meson ratio on the away side.
\end{abstract}

In $\mathrm{Au}+\mathrm{Au}$ collisions at $\mathrm{RHIC}$ an anomalously large number of $p$ and $\bar{p}$ have been observed at intermediate $p_{T}(2-5 \mathrm{GeV} / \mathrm{c})$ in central collisions while the $p / \pi$ ratio in peripheral collisions is similar to $\mathrm{p}+\mathrm{p}$ [1]. Coalescence/recombination models [2, 3, 4] explain this ratio by modeling the production of baryons and mesons in this $p_{T}$ range as coming dominantly from the coalescence of flowing thermal partons from lower $p_{T}$ rather than the fragmentation of a higher $p_{T}$ parton, as is usually assumed. Such models are able to reproduce the observed hadron spectra and the species dependence of the elliptic flow. Angular correlations provide an important means for testing this assumption. If the intermediate $p_{T}$ hadrons are produced by fragmentation they should have associated lower $p_{T}$ particles from the jet fragmentation in a manner similar to what is observed in $\mathrm{p}+\mathrm{p}$ collisions. If they, instead, are produced by coalescence of thermal partons, there should be no jet-like associated lower $p_{T}$ particles. We identify the trigger particle as a baryon $(p$ or $\bar{p})$ or a meson $(\pi$ or $K)$ to investigate whether the excess baryons have a jet-like nature.

The particle composition of the fragmentation products, i. e. lower $p_{T}$ particles associated with jets, provides information about possible modification of the fragmentation process by the hot matter created in central $\mathrm{Au}+\mathrm{Au}$ collisions. By triggering on a leading hadron, a bias is created toward the hard scattering occurring such that the trigger particle traverses very little of the hot matter. Consequently the away side jet at $\Delta \phi=\pi$ has a longer than average path length through the medium. Differences in the particle composition of the associated particles on the away side compared to the near side of the jet provide information about the possible modification of the fragmentation function in the hot medium.

$\S$ For the full PHENIX Collaboration author list and acknowledgments, see Appendix "Collaborations" of this volume. 


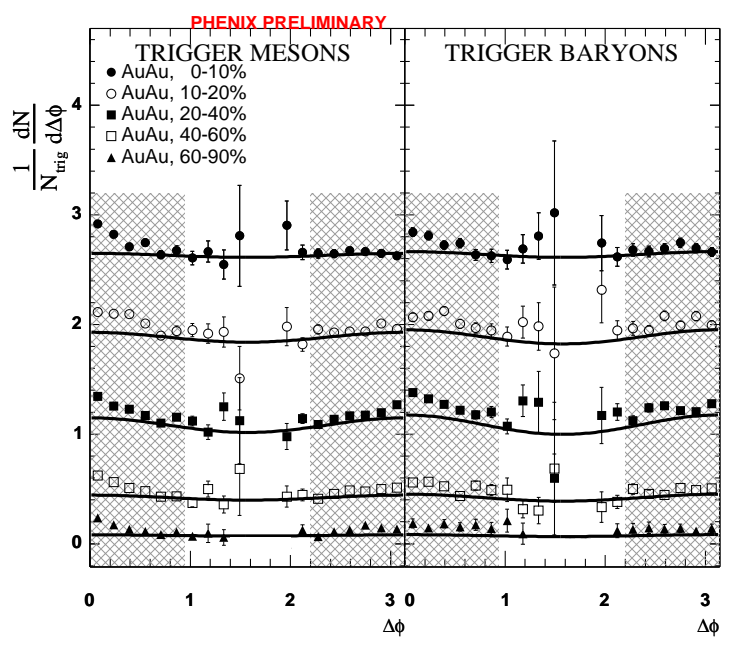

Figure 1. Fully corrected relative angle distributions for identified trigger particles for five centrality bins.

In this analysis 25 million minimum bias events are used. Charged particles are tracked in both PHENIX arms by a Drift Chamber (DC) and Pad Chamber 1 (PC1). Particles are either trigger particles with $2.5<p_{T}<4.0 \mathrm{GeV} / \mathrm{c}$, a matching cut in the PHENIX High Resolution Time of Flight (TOF), and a mass cut identifying it as either a baryon or a meson $\left(\pi\right.$ or $K$ ) or associated particles with $1.7<p_{T}<2.5 \mathrm{GeV} / \mathrm{c}$ and a matching cut in the Pad Chamber 3 (PC3). Particle correlations are studied as a function of $\Delta \phi$, the azimuthal angle difference between trigger and associated particles. The same distribution is made taking trigger and associated particles from different events; the shape of the mixed event distribution provides a measure of the PHENIX azimuthal acceptance for particle pairs, and the absolutely normalized mixed events determine the combinatorial background. The associated particle yield per trigger is determined, correcting for the single track reconstruction efficiency. The resulting $1 / N_{\text {trig }} d N / d \Delta \phi$ distribution has two sources of azimuthal correlations, elliptic flow and jets. Elliptic flow produces an angular correlation of:

$$
B\left(1+2 v_{2}^{\text {assoc }} v_{2}^{\text {trig }} \cos (2 \Delta \phi)\right)
$$

where $v_{2}^{\text {assoc }}$ and $v_{2}^{\text {trig }}$ are the $v_{2}$ values for the associated and trigger particles, respectively, taken from [5]. $B$ is the level of combinatoric background and is taken from the mixed events. The combinatorial background, with the elliptic flow modulation is then subtracted, leaving only jet-like correlations. Figure 1 shows the $1 / N_{\text {trig }} d N / d \Delta \phi$ distributions for five centralities with the solid lines indicating the non-jet correlations. To get the near side (far side) associated particle yield per trigger we integrate the jet correlation $0<\Delta \phi<0.94(2.2<\Delta \phi<\pi)$.

In the second analysis, both particles are charged particles which are tracked in the DC and PC1. Trigger particles have $2.5<p_{T}<4.0 \mathrm{GeV} / \mathrm{c}$, while associated particles have $1.0<p_{T}<2.5 \mathrm{GeV} / \mathrm{c}$ and are identified as either protons/anti-protons or 


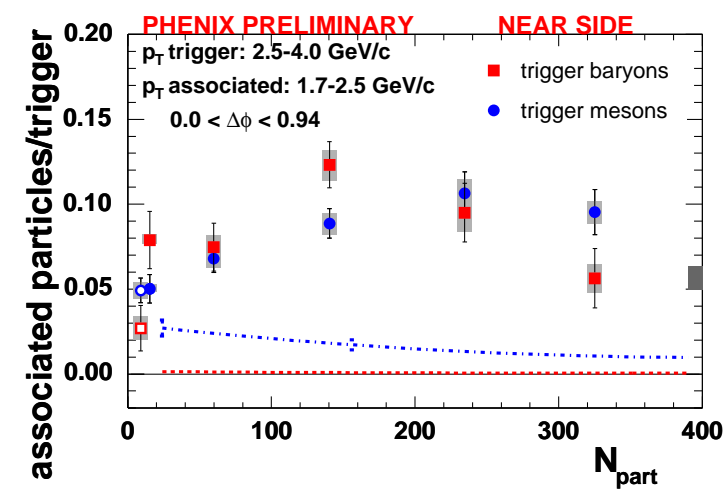

Figure 2. Near side associated particle yield per trigger. Dashed (dot-dashed) line is the prediction for protons (pions) from 2] as described in text. Open points are $\mathrm{d}+\mathrm{Au}$ values.

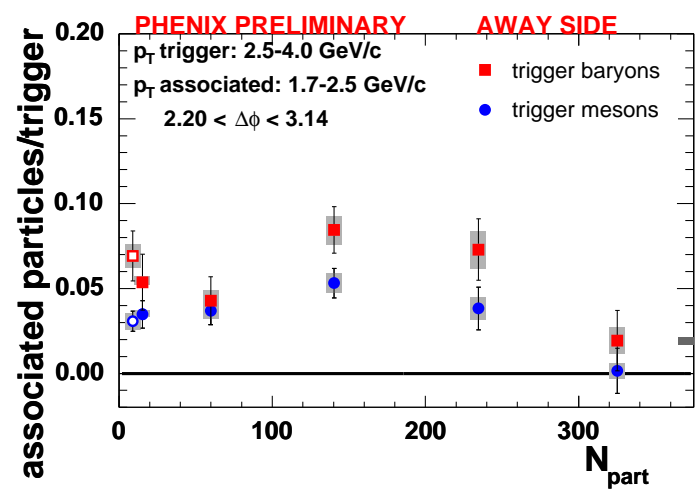

Figure 3. Away side associated particle yield per trigger. Open points are $\mathrm{d}+\mathrm{Au}$ values.

mesons $(\pi, K)$ via timing in the PHENIX Electromagnetic Calorimeter (EMCal). The pairs are used to form traditional correlation functions, with the various contributions deconvoluted via a simultaneous fit. The $v_{2}$ values are obtained by fitting correlation functions made with different orientations of the trigger particle with respect to the reaction plane [6]. With the $v_{2}$ values known it is possible to fit the non-flow part of the correlation function to two Gaussians, one for the near side jet and one for the away side jet. Corrections for the partner efficiency are applied and the absolute associated particle yield per trigger for the near (away) side is the integral of the Gaussian for $0<\Delta \phi<\pi / 2(\pi / 2<\Delta \phi<\pi)$.

Figure 2 shows the near side associated particle yield per trigger for both $\mathrm{d}+\mathrm{Au}$ and $\mathrm{Au}+\mathrm{Au}$ as a function of the number of participating nucleons. There is no decrease in yield going from $\mathrm{d}+\mathrm{Au}$ to central $\mathrm{Au}+\mathrm{Au}$ collisions as would be expected if the triggers (both baryon and meson) were being increasingly produced by the coalescence of thermal quarks. Within statistical and systematic errors, the centrality dependence is not strong.

The fraction of the trigger particle yield arising from recombination is taken from 2]; these triggers should have no jet-like partners. The remaining triggers are assigned a partner probability equal to that measured in $\mathrm{d}+\mathrm{Au}$, shown in the figure by the open circles. The combined partner yield per trigger is shown by the lines, and clearly underpredicts the data for all $\mathrm{Au}+\mathrm{Au}$ centrality selections. The comparison indicates that the picture is incomplete, and it has been suggested to include also recombination between thermal partons and shower partons created as jets fragments [4, 3].

Figure 3 shows the same quantity for the away side jet. We see a decrease in the yield going from $\mathrm{d}+\mathrm{Au}$ to central $\mathrm{Au}+\mathrm{Au}$ which is in agreement with previous measurements of away side suppression/broadening [7, 8]. 


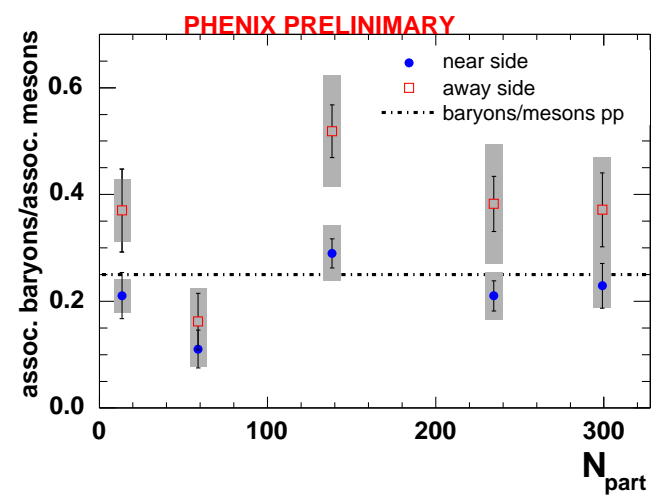

Figure 4. Baryon to meson ratio of jet fragments. Dashed line indicates the baryon to meson ratio from the single particle spectra in $\mathrm{p}+\mathrm{p}$ at the $\left\langle p_{T}\right\rangle$ of the jet fragments.

Figure 4 shows the result of the analysis of identified associated particles. The baryon to meson ratio for associated particles in the same jet as the trigger particle and the opposing, or away-side jet, are plotted as a function of the number of participants. The dashed line shows the baryon to meson ratio of the single particle yields in $\mathrm{p}+\mathrm{p}$ collisions at the $p_{T}$ corresponding to the mean $p_{T}$ of the associated particles. The nearside ratio is consistent with $\mathrm{p}+\mathrm{p}$, but the away-side ratio is higher. Studies are currently underway to quantify any possible effects of the PHENIX pseudorapidity acceptance upon this ratio, but the data may be showing a modification of the jet fragmentation by the medium.

In summary, we have shown azimuthal correlations of identified jet fragments. The identified trigger particle analysis shows that intermediate $p_{T}$ meson and baryon production is not dominated by the coalescence of thermal partons. Other models, which allow coalescence of thermal partons with those arising from fragmentation, may more closely reproduce the data, as a larger fraction of intermediate $p_{T}$ hadrons would be predicted to have jet-like partners. Identifying the away-side partners offers a way to control the path length in the medium and help constrain the models.

\section{References}

[1] PHENIX Collaboration Adler S S et al. 2003 Phys. Rev. Lett. 91172301

[2] Fries R J, Müller B, Nonaka C and Bass S A, 2003 Phys. Rev. C 68044902

[3] Hwa R and Yang C B 2004 arXiv:nucl-th/0401001

[4] Greco V, Ko C M and Lévai P, 2003 Phys. Rev. Lett. 68034904

[5] PHENIX Collaboration Adler S S et al. 2003 Phys. Rev. Lett. 91182301

[6] Bielcikova J et al. 2004 Phys. Rev. C 69021901

[7] STAR Collaboration Adler C et al. 2003 Phys. Rev. Lett. 91082302

[8] Rak J for the PHENIX Collaboration, these proceedings.

[9] Matathias F for the PHENIX Collaboration, these proceedings. 